\title{
Karyologisch-systematische Untersuchung der Tribus Loteae und Phaseoleae Unterfam. Papilionatae
}

\author{
Von WL. TSCHECHOW und N. KARTASCHOWA \\ Aus dem Kabinet für Zytologie und Selektion \\ an der Staats-Universität zu Tomsk
}

Eingegangen an ro. Dezember $193^{\prime}$

Die in unserem Laboratorium begonnene Untersuchung der Unterfamilie Papilionatae nähert sich dem Ende.

Die Untersuchung der Tribus Trifolieae DC., Galegeae BronN., Sophoreae Sprengel, Podalirieae Benth., Genisteae Bronn., Hedysareae DC., Loteae BENTH. und Phaseoleae BroNN. ist beendigt.

Die erhaltenen Ergebnisse haben als Material zu 6 Arbeiten gedient, von welchen schon 3 gedruckt sind und 2 zum Druck vorbereitet werden.

Abgesehen davon, da $\beta$ die Untersuchung von Tribus Vicieae BRoNN. im Jahre 1926 begonnen wurde und Chromosomenzahlen ungefähr für 60 Arten vorhanden sind, wird sie nicht früher als in einem halben Jahre beendigt werden. Die Chromosomenzahlen für diese Tribus geben zu wenig. Es ist ein ausführlicheres Studium des Idiogrammes nötig.

Die gegenwärtige Untersuchung, die zwei Tribus umfaßt, hat vor allem den Zweck, eine karyologisch-systematische Charakteristik auf Grund der Chromosomenzahlen zu geben. Obgleich die Chromosomenzahlen doch nicht alle jene komplizierten Veränderungen, welche das Idioplasma erfährt, abspiegeln, helfen sie doch in manchen Fällen eine der komplizierten Fragen der Biologie aufzulösen-,, die Wege der Organismenevolution anzudeuten." Als beste Illustration dieser Thesis kann die Arbeit von N. P. AvduLow über die Frage der karyologischsystematischen Analyse der Familie Gramineae dienen.

Beim Studium der Tribus Loteae der Gattung Lotus und der Tribus Phaseoleae bei Clitoria ternatea hielten wir für möglich eine genauere Analyse der karyologischen gegenseitigen Verhältnisse vorzunehmen 
durch Feststellung der Chromosomenindividualität. Gegenwärtig kann die Bestimmung der Chromosomenzahlen allein die Zytologen nicht befriedigen, in den Arbeiten von Nawaschin, LewItsky, DeLonay, SWETSCHNIKOWA u.a. Autoren sind die Idiogramme einzelner Arten genau studiert. In einer der letzten Arbeiten, die ihren Resultaten nach sehr interessant ist (von SENJANINOWA-KoRTSCHAGINA ,,Karyologisch-systematische Forschung der Gattung Aegilops L." Berichte der U.d.S.S. R., Tagung für Genetik und Selektion, Leningrad den 10-16 Januar 1929 B. II., Genetik), finden wir nicht nur Anweisungen auf die Hybriden-Natur von Aegilops persica und Aegilops cylindrica HosT, sondern auch eine genaue Bestimmung, aus welchen Arten die gegebenen Arten

Ae. umbelulata $\times$ Ae. caudata BeRT. $\rightarrow$ Ae. persica

Ae. bicornis BoIss. $\times$ Ae. caudata $\rightarrow$ Ae. cylindrica Host.

entstehen konnten.

Mittels der Chromosomenindividualisierung und einer genauen $\mathrm{Zu}$ sammenstellung des Idiogrammés, können wir unsere Möglichkeiten im Sinne einer weiten Anwendung der zytologischen Methode in der Systematik und Genetik ausdehnen. Aber wie verlockend auch diese Perspektiven sind, müssen wir dennoch konstatieren, daß bis zur letzten Zeit diese Art zytologischer Untersuchungen eine sehr beschränkte Zahl der dankbarsten Arten umfasste. Deswegen sind die Gattungen Muscari, Galtonia, Crepis, Bellevalia ein Lieblingsobjekt der klassischen Untersuchungen der Zytologen verschiedener Länder geworden. Trotzdem, daB das Prozent der zytologisch untersuchten Arten bedeutend wächst und mit jedem Jahre zunimmt, bleibt die Zahl der Organismen wie früher, die zur Feststellung eines genauen Idiogrammes bequem sind, sehr klein.

Die Möglichkeit, die Zahl der in zytologischer Hinsicht günstigen Arten zu vermehren, hängt, wie es scheint, in vielem nicht nur von den durch uns untersuchten Organismen, sondern auch von jenen Methoden, die wir beim Untersuchen anwenden, ab. Diese Thesis ist glänzend durch eine Reihe von Arbeiten des Laboratoriums von Prof. G. A. Lewitsky bewiesen.

Das von G. A. LEwITSKY in verschiedenen Modifikationen angewendete chondriosome Fixiermittel ${ }^{1)}$ hat in Beziehung auf Bohnenarten keine genügend gute Resultate gegeben, aber ohne Zweifel, wird man

1) $10 \%$ Formalin $1 \%$ Chromsäure. 
auch für diese Pflanzengruppe später ein besseres Fixiermittel, anstatt der gegenwärtig existierenden finden können.

Aber wenn die Vervollkommnung der Methodik der Zubereitung von Präparaten unsere Möglichkeiten erweitert, so bleibt immerhin noch ein schwer zu überwältigendes Hindernis : eine beträchtliche Anzahl von Organismen hat der GröBe nach unbedeutende Chromosomen, und indem wir sie sogar beim Vorhandensein klar ausgedrückter Segmentation studieren, ist es unendlich schwer nicht nur ein Quantitätschema zu konstruieren, sondern auch wechselseitige Beziehungen zwischen den Schultergrößen festzustellen und ihre Gruppierung der Größe nach durchzuführen.

Unsere Untersuchung ist ein Versuch der Feststellung von Chromosomenindividualität beim Studium der kleinen Chromosomenarten. Die Vertreter der Tribus Loteae bei Anwendung des Fixiermittels von NAWASCHIN, bei einer gebührenden Differenzierung und Durchsichtigkeit der Präparate, die in unserem Laboratorium durch Aufklärung mit Nelkenöl und Xylol erreicht wird, erscheinen im Sinne der Segmentation als ein genügend günstiges Material.

Den Aufbau eines quantitativen Schemas haben wir infolge der geringen Chromosomendimensionen sofort zurückgewiesen und hielten es für möglich die Größe nicht in absoluten Einheiten auszudrücken, sondern bezüglich : kleine, mittlere, große, gleichschulterige, ungleichschulterige Chromosomen usw. In der Absicht der gröBten Sicherheit und der Ausnahme irgend welcher Voreingenommenheit wurden die Präparate so studiert, daß der Forscher die Stellung im System der von ihm studierten Art nicht kannte. Die Verteilung der Chromosomen in große, mittlere und kleine und innerhalb jeder von diesen Gruppen in Beziehung auf Schultern wurde von 2 Personen unabhängig von einander untergenommen. Nachdem wurden die Präparate zum 2-ten Mal durchgesehen, und die karyologischen Ergebnisse wurden mit den systematischen Kennzeichen und Aufbauungen zusammengestellt. In allen Fällen stimmten die Ergebnisse, die zur Gruppe ,,große Chromosomen" gehörten, ganz überein, doch in Beziehung auf die 2 anderenerwiesen sich Verschiedenheiten, nicht nur zwischen beiden Forschern, sondern selbst bei einem und demselben, bei einer Zusammenstellung der Ergebnisse der ersten und zweiten Durchsicht.

Vor allem betrafen diese Verschiedenheiten die Verteilung der Chromosomen in Gruppen von kleinen und mittleren und außerdem auch in der Korrelation von Schultern bei kleinen Chromosomen. Die Teilung der Chromosomen der Größe nach ist konventionell, doch beim 
Studium der Arten der Gattung Lotus erwirbt diese Konvention noch größere Dimensionen, da es keine scharfe Sprünge der Größe nach gibt und beim Vorhandensein fließender Übergänge es schwer ist eine Grenze durchzuführen.

Am leichtesten ist es eine Gruppe von großen Chromosomen auszusondern. Aber wenn diese Aufgabe für jede Art im Einzelnen zu realisieren ist, so sehen wir, indem wir die Zeichnungen und Resultate der Gruppierung bei verschiedenen Arten zusammenstellen, da $B$ die Chromosomen einer Art, die der Gruppe von "mittleren" zugeschrieben sind, nicht größer als die Chromosomen einer anderen Art sind, die der Gruppe von "kleinen" zugeschrieben sind und umgekehrt. Die oben angewiesenen Schwierigkeiten veranlaßten uns den Aufbau der Gesamttabelle zu verändern, indem die Ergebnisse, die zu den großen Chromosomen gehören, in sie eingeführt werden, die mittleren und die kleinen in eine Gruppe vereinigt und in ihr die weiteren, Gliederungen der Schulterngröße nach, nur in den Fällen, wo das sicherer erscheint, durchgeführt werden. Doch beim Beschreiben einzelner Idiogramme sind ausführlichere Kommentare mit Anweisung der wahrscheinlichsten Gruppierungen und der gefundenen Schwierigkeiten angeführt.

Wenn beim Studium der großen Chromosomenarten die Orientierung (die Neigung des ganzen Chromosomen und der einzelnen Schultern) mit Hilfe einer mikrometrischen Schraube genau berechnet werden kann, so ist das bei kleinen Chromosomen bedeutend schwieriger zu machen.

Bei karyologisch-systematischen Untersuchungen der großen taxonomischen Einheiten-der Familien strebt man die größte Anzahl von Gattungen zu umfassen, indem je 2-3 Vertreter von jeder Gattung studiert werden. Ein solches Material gibt immerhin genügende Auskünfte für die Charakteristik jeder Gattung, bezüglich der Grundzahlen von Chromosomen und dem Vorhandensein von Polyploidie. Doch beim Studium der Gattungen strebt man die größte Anzahl von Arten zu umfassen. Beim Studium der Familie Leguminosae beschränkten wir uns jedoch nicht mit einer kurzen Gattungscharakteristik und soviel das Material erlaubt, versuchten wir unsere Untersuchung auf eine größere Anzahl von Arten zu erweitern, um auch innerhalb der Gattung phylogenetischen Aufbau zu planen.

Die unten angeführten karyologischen Beschreibungen sind auf dem Grunde der besten Platte gemacht, aber Chromosomen, die sich am schärfsten durch ihre individuellen Eigenheiten unterscheiden, sind in allen vorhandenen Metaphasen verfolgt. 
Die Methodik der Zubereitung von Präparaten und das Aufzeichnen bleiben allgemein für alle unsere Untersuchungen :

das Fixiermittel von NAWASCHIN

$\begin{array}{cr}\text { 1\%-ige Chromsäure } & 10 \text { Teile } \\ \text { 10\%-iges Formalin } & 4 \text { Teile } \\ \text { Eis-Essigsäure. } & 1 \text { Teil. }\end{array}$

Die Färbung mit Eisenhämatoxylin nach HEIDENHAIN.

Die Zeichnungen sind mittels des Zeichenapparates ABBÉ bei 3250 facher Vergrößerung in Höhe des Tisches ausgeführt (das Immersionssystem Apochromat ZEISS $2 \mathrm{~mm}$ und Kompensationsokular ZEISS 20). Für Clitoria ternatea bei 2250 facher Vergrößerung.

\section{Karyologisch-systematische Charakteristik}

Es ist nicht möglich eine Charakteristik den Tribus der Unterfam. Papilionatae zu geben, indem wir auf die Kennzeichen die für alle Vertreter einer Tribus allgemein sind und die sie von allen anderen Tribus unterscheiden, hinweisen.

,, 10 freie Staubfäden “ sind ein charakteristisches Merkmal auch für Sophoreae wie für Podalirieae.

,, 10 Staubfäden, meistenteils verbundene, seltener 1 freier und 9 zusammengewachsene "-werden bei den Tribus Genisteae und Podalirieae beobachtet und endlich ,, 10 Staubfäden, seltener alle verbunden, öfter ein freier und 9 verbundene " werden bei allen übrigen Tribus: Trifolieae, Loteae, Galegeae, Hedysareae, Vicieae, Phaseoleae gefunden. Es ist ziemlich schwierig die Tribus zu charakterisieren, indem auch die Kennzeichen, die zu den vegetativen Organen gehören, benutzt werden.

Tribus Loteae schließt 8 Gattungen ein : Anthyllis L. (20-30 A.), Dorycnium L. (10-12 A.), Hymenocarpos SAvi.= Circinnus MEDIK. (1 A.), Lotus L. (80-90 A.), Securigera DC.=Bonaveria Scop. (1 A.), Cytisopsis JAUB. et SPACH. (1 A.), Helminthocarpum A. RICHE. (1 A.) und Hosackia DoUGL. (30 A.).

Die ersten 5 Gattungen sind der Gegenstand unserer Untersuchung ; Cytisopsis, der in Syrien und Sizilien gefunden wird, Helminthocarpum, der in Abyssinien wächst und ebenfalls die Nordamerikanische Gattung Hosackia sind noch nicht von uns wegen der Abwesenheit von Materialien, studiert.

Als phytogeographische Charakteristik der von uns studierten Tribus kann die unten angeführte Tabelle, die nach den Materialien der Arbeit von CAPITAINe (,, Étude analytique et phytogéographique 
du groupe des Légumineuses ", Bul. de Géogr. botanique, Bd. XXIII, 1913), gebildet ist, dienen.

Anstatt einzelne Tabellen für jede uns interessierende Tribus zu geben, hielten wir für möglich eine gesamte Tabelle zusammenzustellen, wodurch wir eine größere Anschaulichkeit erreichen.

Wenn wir die obenangeführte Tabelle betrachten, sehen wir, daß Tribus ,Loteae" vor allem die Formen, die am meisten im Gebiete des mittelländischen Meeres verbreitet sind, einschließt und außerdem sind 90\% aller Arten nur in Europa, Asien und Afrika zu finden.

Die in der Tabelle für Nord-Amerika von CAPITAINE angeführten Arten gehören zur Gattung Hosackia; außer den Vertretern dieser Art sind keine andere Gattungen von Tribus Loteae in Nord-Amerika vorhanden.

Es ist interessant hervorzuheben, daß die Arten von Tribus Trifolieae ebenso wie von Tribus Loteae sich über die Kontinente verbreiten; das größte $\%$ ist in Eurasien und das geringste in Amerika vertreten.

Wie Loteae findet man die meisten Gattungen und Arten der Tribus Trifolieae im Gebiete des mittelländischen Meeres, aber ihr Areal ist doch breiter und sie umfassen eine größere Fläche des Erdballs.

Nach den Ergebnissen, die in unserer Literatur vorhanden sind, im Gegensatz zu den anderen Tribus der Unterfamilie „Papilionatae,“ ist Tribus , Loteae " noch durch niemand zytologisch studiert.

\section{Karyologisch-systematische Charakteristik der Gattungen} Anthyllis, Hymenocarpos, Securigera und Doricnium

Zur Gattung Anthyllis L. gehören 20-30 Arten, die in 7 Sektionen von TAUBERT geteilt werden.

Die west-mittelländische Sektion Aspalathoides DC. und die Sektion Dorycnioides DC. und Cornicina BoIss., deren Vertreter in Spanien und Portugal gefunden werden, sind von uns nicht studiert.

Sektion Vulneraria DC.

$\mathrm{Zu}$ dieser Sektion gehören 3 Arten, die in ganz Europa und Afrika weit verbreitet sind; 2 Arten der sehr polymorphen kollektiven Art von Anthyllis Vulneraria DC. sind von uns studiert worden.

A. maritima (Abb. 1)-2 Chromosomen (a) unterscheiden sich bedeutend der Größe nach von den übrigen, indem sie sie der Größe, nach ungefähr $2 \mathrm{Mal}$ überragen. Aus 10 kleinen zweischulterigen Chromosomen können 2 Chromosomen mit Köpfchen ausgesondert werden (r). 


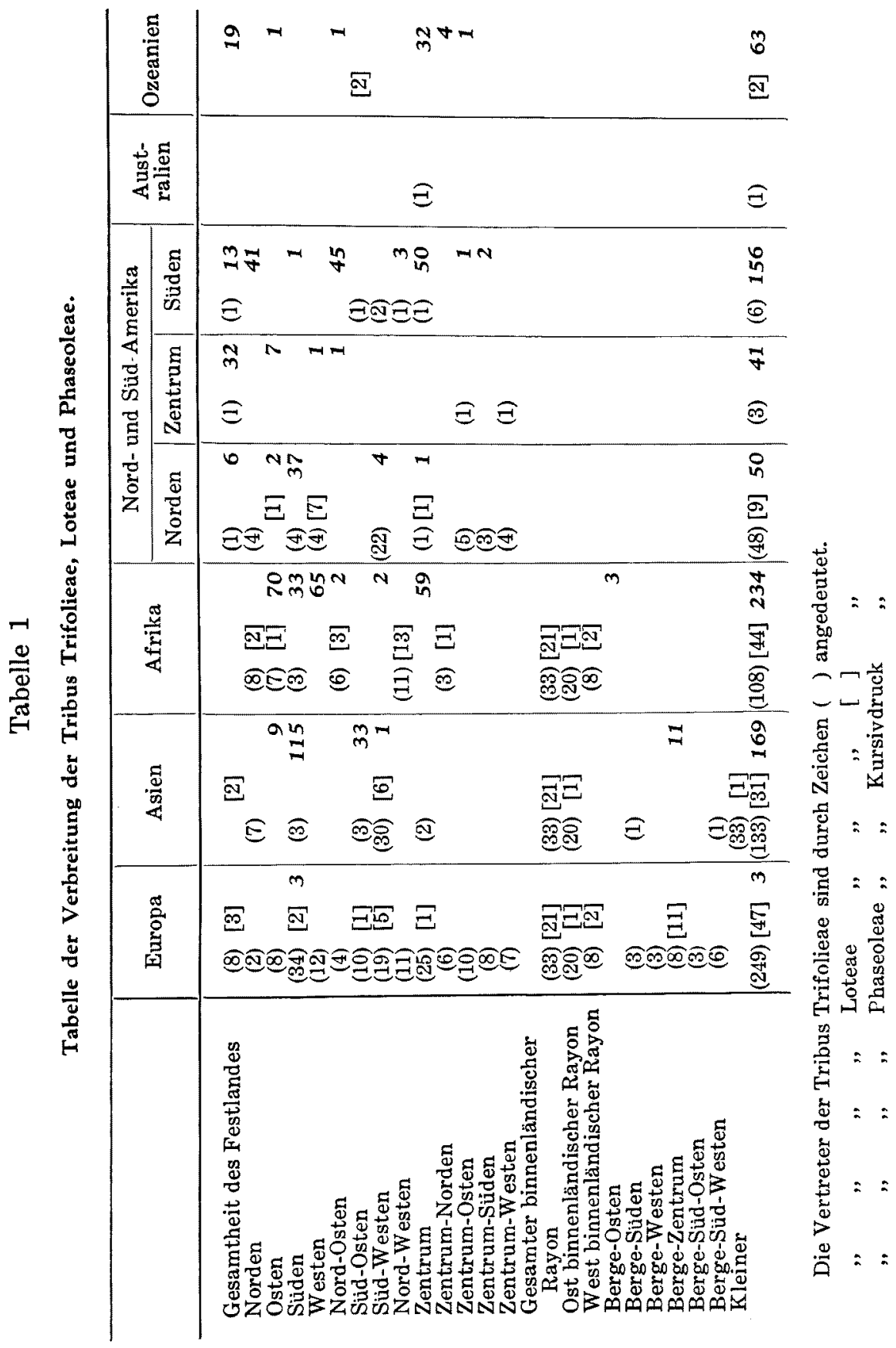


Wie bei der vorhergehenden Art findet sich bei A. alpestris (Abb.2), ein Unterschied in der Größe der Chromosomen.

4 sehr kleine Chromosomen (k) und 2 Chromosomen mittlerer Größe $\left(k_{1}\right)$ schreiben wir der Gruppe von mittleren und kleinen Cbromosomen zu. Zwischen 6 großen Chromosomen tragen 2 Chromosomen (f) die allergrößten, je einen Trabanten.

Die Sektion Oreanthyllis GRISEB hat die meiste Zahl von Arten (10), die im mittelländischen Gebiete wachsen.

Von uns ist A. Barba Jovis L. (Abb. 3) studiert. 14 Chromosomen dieser Art kann man in 2 Gruppen teilen-8 sehr große, ungleichschulterige (b) und 6 geringere der Größe nach, gleichschulterige (k).

Die Sektion Dorycniopsis LEM. enthält nur eine Art-A. Gerardi L. (Abb. 4), mit 16 Chromosomen deren Areal das Gebiet des mittelländischen Meeres ist.

Die Chromosomen dieser Art unterscheiden sich nicht nur ihrer Größe nach, sondern auch durch ihre individuellen Eigenheiten. Es zeichnen sich scharf 4 Chromosomen mit großen Trabanten (x) und 2 klar dreischulterige Chromosomen (c) aus. Alle übrigen 10 Chromosomen sind zweischulterig, 6 von ihnen, ungleich zweischulterig, kann man auf Grund der Beziehung der Schulterlänge in 3 Paare zerteilen : das erste Paar mit der längsten kurzen Schulter $\left(b_{1}\right)$, das zweite mit einer kürzeren $\left(b_{2}\right)$ und endlich das dritte Paar mit einer noch kürzeren Schulter $\left(b_{3}\right)$.

Bei vier gleichschulterigen Chromosomen ist die Gliederung weniger sichtbar, zwei von ihnen sind bedeutend kleiner (k) und zwei viel größer $\left(\mathrm{k}_{1}\right)$.

Zur Sektion Physanthyllis BoIss. gehört ein im ganzen mittelländischen Gebiete weit verbreiteter $A$. tetraphylla L. (Abb. 5). Es ist uns nicht gelungen eine Gruppierung von 16 Chromosomen dieser Art durchzuführen, trotz einer großen Anzahl von Metaphasen, da die Segmentation der Chromosomen ziemlich schwach ausgedrückt ist und keine Platten, wo sie in einer Fläche gelagert wären, vorhanden waren.

Die Gattung Hymenocarpos SAvI., die im ganzen Gebiet des mittelländischen Meeres zu finden ist, ist nur durch eine Art $H$. circinnatus SAVI, (Abb. 6.), die 16 große zweischulterige Chromosomen hat, dargestellt. Leider, is es schwer eine endgültige Gruppierung dieser Chromosomen durchzuführen. Wahrscheinlicher ist, daß 6 Chromosomen ungleichschulterig sind, von den übrigen 10 gleichschulterigen Chromosomen sind zwei etwas kleiner. 
Zur Gattung Securigera DC. gehört nur eine Art, die wie alle anderen Vertreter der Tribus Loteae im Gebiete des mittelländischen Meeres gefunden wird. Es ist sehr schwer, die Chromosomen bei $S$. Coronilla DC. zu gruppieren. Sie sind alle (12) zweischulterig, wobei die Korrelation der Schultern von 3:2 vorherrscht. Zwei-drei Paar der Chromosomen kann man den gleichschulterigen zuschreiben. Indem wir eine bedeutende Anzahl von Präparaten und sehr bequeme zum Zusammenzählen der Metaphase haben, fanden wir keinen Fall, wo die Chromosomen in einer Fläche waren, die Neigung der Chromosomen (die in einzelnen Fällen $45^{\circ}$ erreicht) entzieht uns die Möglichkeit die Schulterlänge zu bestimmen (Abb. 7).

Die Vertreter der Gattung Dorycnium L. werden beinahe ausschlieBlich im Gebiete des mittelländischen Meeres gefunden. Die Chromosomen der Größe nach beim Vertreter dieser Gattung zu gruppieren, ist bedeutend leichter.

Wie man auf der Abbildung (8) sieht, sind die 14 Chromosomen von D. hirsutum SER. der Größe nach ungleich. Es zeichnen sich scharf zwei sehr lange dreischulterige Chromosomen aus (c) und vier kleine gleichschulterige (k). Von den übrigen zweischulterigen Chromosomen sind 4 etwas größer (a).

Bei D. rectum SER. werden auch scharfe Unterschiede in der Größe der Chromosomen beobachtet (Abb. 9). Von der Gesamtzahl der 14 Chromosomen sind 8 am größten, 2 gleichschulterig (a), 6 ungleichschulterig $(\mathrm{N}, \mathrm{b})$. Wobei die Chromosomen $(\mathrm{N})$ etwas kleinere Länge haben. Die gebliebenen 6 Chromosomen sind geringer, sie nehmen allmählich der Größe nach ab (k).

Die Zahl der Chromosomen von D. suffruticosum VILL. ist 14 (Abb. 10). Von den größten Chromosomen sind 4 beinahe gleichschulterig (a) und 2 Chromosomen ungleichschulterig (b). Alle übrigen Chromosomen werden von uns der Größe nach zu den mittleren und kleineren angerechnet. Von ihnen können 2 Chromosomen mit kürzerer zweiter Schulter abgesondert werden (N).

Wir gehen zum letzten Vertreter der Gattung Dorycnium: D. herbaceum VILL. über (Abb. 11).

Hier finden wir wieder große dreischulterige Chromosomen (c) vor, ihre Zahl ist 4, während bei D. hirsutum SER. ihrer nur 2 waren. Diese Art unterscheidet sich scharf von allen ubrigen Dorycnium wegen der Anwesenheit von 6 ungleichschulterigen Chromosomen mit sehr kurzer zweiter Schulter. Zwei von ihnen (d), etwas größere, werden von uns als große Chromosomen betrachtet, 4 Chromosomen (r) sind auf unserer 


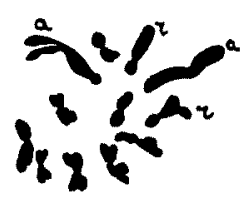

1
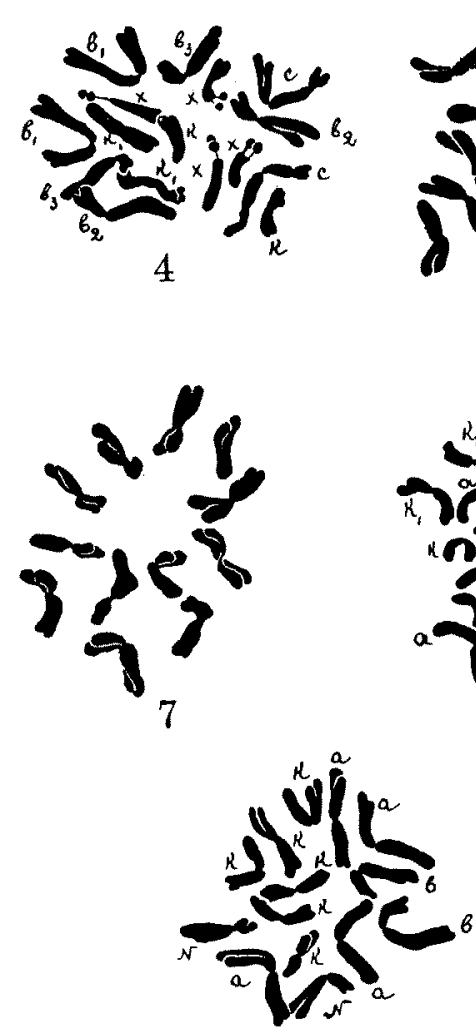

10

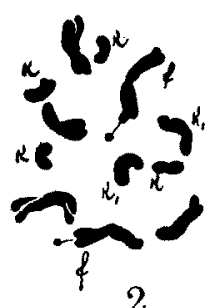

2

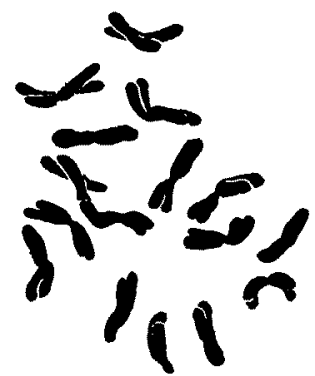

5

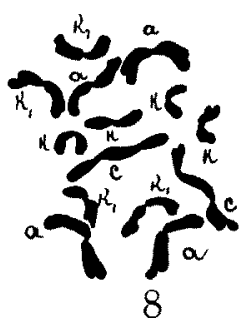

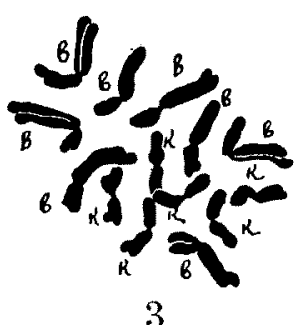
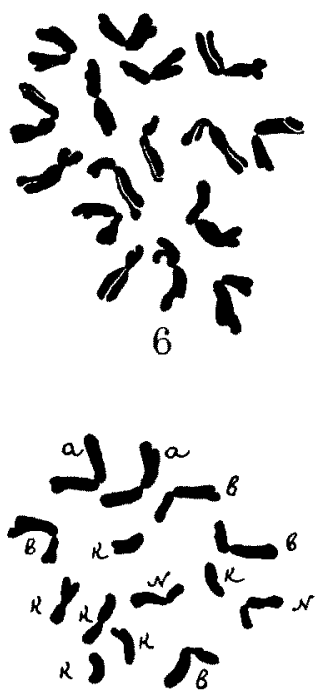

9

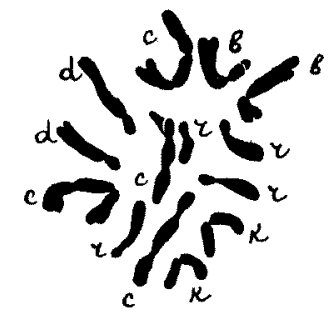

11

Abb. 1-11

1. Anthyllis maritima ScHweigg.

2. A. alpestris KIT.

3. A. Barla Jovis L.

4. A. gerardi L.

5. A. tetraphylla L.

6. Hymenocarpos circinnatus SAVI.
7. Securigera coronilla DC.

8. Dorycnium hirsutum SER.

9. D. rectum SER.

10. D. suffruticosum VILL.

11. D. herbaceum VILI. 
Tabelle zu den mittleren und kleinen Chromosomen angerechnet. Es bleibt nur von 4 Chromosomen zu sprechen -2 Chromosomen sind am geringsten, gleichschulterig ( $\mathrm{k}$ ) und 2 Chromosomen ungleichschulterig, groß (b). Alles obenerwähnte ist in folgender Tabelle 2 zusammengeführt:

\section{Karyologisch-systematische Charakteristik der Gattung Lotus L.}

Vom Gesichtspunkte der Systematik aus, ist die Gattung Lotus genauer studiert worden. BRAND trägt Veränderungen in die früher existierende Teilung der Gattung Lotus in Sektionen: 1) Krockeria, 2) Loteae, 3) Eulotus, 4) Microlotus, 5) Ononidium ein ; die Gattung Lotus teilt sich in 2 Untergattungen.

Die Untergattung Pedrosia (Sektionen : a) Heinekenia-dessen einziger Vertreter L. peliorrhynchus ist und b) Eupedrosia) hat eine sehr beschränkte Verbreitung (West-Afrikanische Inseln) und ist in unserer Untersuchung nicht dargestellt.

Die zweite Untergattung $E$ dentolotus wird durch BRAND in folgende Sektionen geteilt: Krockeria, Xantholotus und Erythrolotus (anstatt Loteae und Eulotus) : Ononidium und auBerdem L. tetraphyllis wird in eine besondere Sektion Quadrifolium abgeteilt.

Die Sektion Microlotus ist von Taubert (Engler und Prantl „, Die natürlichen Pflanzenfamilien ") und BRAND den Arten der Gattung Hosackia zugeschrieben. Die Vertreter dieser Sektion und der ganzen Gattung Hosackia treten nicht aus den Grenzen von Nord-Amerika hinaus. Die Arten der Gattung Lotus jedoch nach den Ergebnissen der beiden obenangewiesenen Autoren sind in Europa und Asien hauptsächlich im Gebiete des mittelländischen Meeres und nur wenige in Süd-Afrika und in Australien verbreitet.

Nach der Arbeit von CAPITAINE jedoch werden Vertreter der Gattung Lotus auch in Nord-Amerika, Europa, Asien, Afrika, Australien, Nord-Amerika und West-Australien getroffen.

ASCHERSon und GRAEBNER (Synopsis der Mitteleuropäischen Flora) halten sich der Ansicht von BRAND, wie hinsichtlich der Verbreitung dieser Gattung, so auch hinsichtlich der Einteilungen. Es bestehen nur Veränderungen darin, daß L. Tetragonolobus L. von AsCHERSON und GRAEBNER nicht als eine einzelne Gattung von Tetragonolobus purpureus, sondern nur als eine Untergattung der Gattung Lotus betrachtet ist und zu dieser Untergattung gehört noch L. siliquosus. 


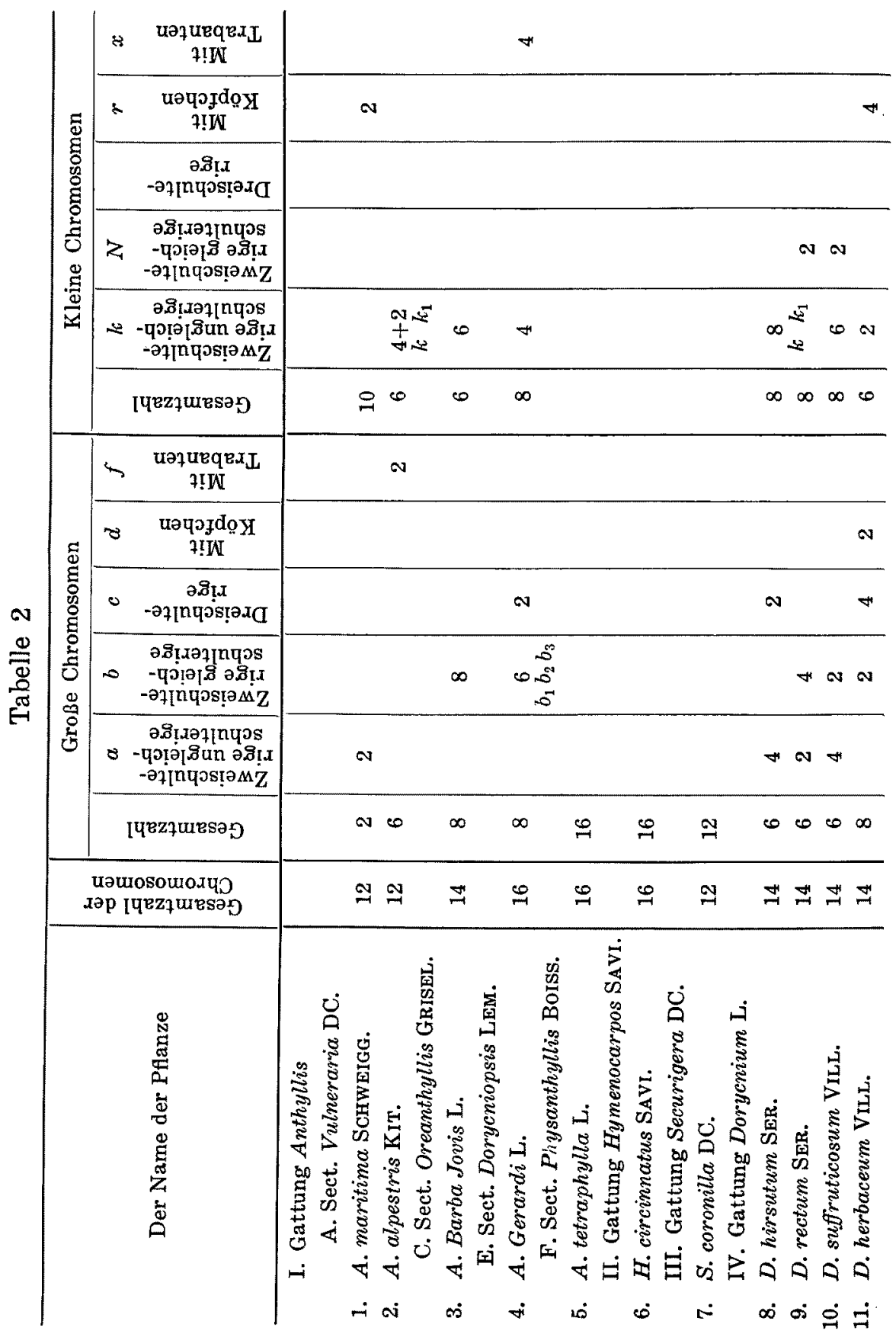


Diese Veränderungen führen auch wir ein. Eine Zahl von ungefähr 100 Arten wird bei ASCHERSON und GraebNer für die Gattung Lotus angeführt, nach den Ergebnissen von CAPITAINE und ENGLER 80-90.

In unserer Untersuchung sind aus der Zahl der obenerwähnten Untergattungen und Sektionen folgende dargestellt: Untergattung Pedrosia Sect. Eupedrosia, Untergattung Edentolotus Sect. Xantholotus, Untergttung Tetragonolobus.

L. Requieni MAURI. wird von BRAND als Tetragonolobus Requieni, Fisch. et MEY. betrachtet, wir aber, Ascherson und Graebner folgend, schreiben ihn der Gattung Lotus der Untergattung Tetragonolobus zu.

Die 14 Chromosomen von L. ornithopodioides (Abb. 12) kann man in 2 Gruppen zerteilen: größere Chromosomen, ungleichschulterige, deren Zahl 4 (a) ist, ihre kurzen Schultern nähern sich dem Köpfchen und Chromosomen geringerer Größe, die Korrelation der Schultern variiert bei der letzten Gruppe und es ist schwierig eine Gruppierung unter ihnen durchzuführen.

L. Creticus (Abb. 13) hat 28 Chromosomen. Trotz der auf den ersten Anblick scheinenden Einförmigkeit der Chromososomen, kann man bei ausführlichem Studium folgende 4 Gruppen feststellen.

I Gruppe:-Gleichschulterige Chromosomen von den geringsten Dimensionen, $-\ldots . .8$ Chromosomen (k).

II Gr.-zweischulterige Chromosomen $1 \frac{1 / 2}{2}$ Mal größer der Dimension nach als die vorhergehenden,.....8 (a). Die Segmentation ist so klar ausgedrückt und sie sind überall so orientiert, daß sie wie zwei geringe, einschulterige, etwas gebogene, mit ihren Enden verbundene, scheinen.

III Gr. - ....4 Chr. (r), die sich der Größe nach, zur ersten Gruppe nähern und vielleicht sogar etwas geringerer Dimension sind-mit Köpfchen versehen, und endlich.

IV Gr. $-\ldots \ldots$. 8 Chromosomen (d) mit sehr kurzer Schulter, der Größe nach nehmen sie (nach der Gruppe IIa) den zweiten Platz ein.

Von 14 Chromosomen des L. cytisoides (Abb. 14) sind 4 Chromosomen (d) gleich wie bei L. ornithopodioides, ungleichschulterig, mit kurzer, sich dem Köpfchen nähernden Schulter. Außerdem zeichnen sich scharf 2 dreischulterige Chromosomen (c), die größten der Dimension nach, aus. Ihre Anwesenheit unterscheidet diese Art nicht nur von den vorhergehenden, die zur Untergattung Edentolotus, die von uns untersucht wird, angehören, sondern auch von allen anderen Arten der Gattung Lotus. Die Gruppierung der übrigen Chromosomen 
bei $L$. cytisoides stellt einige Schwierigkeiten vor, es ist wahrscheinlicher, daß 2 von ihnen gleichschulterige Chromosomen von der geringsten Dimension (k) sind und 6 Chromosomen (4 ungleichschulterige Chromosomen $\left(k_{1}\right)$ der Größe nach eine mittlere Stellung einnehmen.

Jetzt gehen wir zur Gesamtart von L. corniculatus, die eine Grundzahl von 12 Chromosomen hat, über. Der erste von den studierten Vertretern der obenerwähnten Gesamtart von L. uliginosus (Abb. 15.) hat 12 Chromosomen. Auf Grund der Korrelation der Schulterlänge kann man folgende Einteilung durchführen: vier Chromosomen $(3+y)$ etwas kleinere ihrer Dimension nach, mit Köpfchen, wobei das Chromosom (y) nicht ganz identisch homolog für die übrigen drei ist und sich von ihnen durch etwas größere Dimensionen unterscheidet. Die übrigen 8 Chromosomen ist es richtiger in folgende Gruppen einzuteilen:

2 ungleichschulterige Chromosomen mit sehr geringer zweiter Schulter (N) und 6 gleichschulterige Chromosomen ; der Größe nach sind sie verschieden : 1 Paar (a) von der größten Dimension, 1 Paar von der geringsten ( $k$ ) und ein Paar nimmt eine Zwischenstellung ein $\left(k_{1}\right)$.

L. corniculatus ist durch 2 Abarten var. alpestris (Abb. 16) und var. tenuifolius vorgestellt (Abb. 17).

Die erste von ihnen hat 24, die zweite 12 Chromosomen.

Der Größe und der Korrelation der Schultern nach sind die Chromosomen von $L$. corniculatus var. alpestris noch lange nicht gleichartig, hier finden wir 4 gleichschulterige größte Chromosomen (a), 12 Chromosomen geringerer Dimension, mehr oder weniger gleichschulterige und 8 Chromosomen mit Köpfchen.

Die zweite Abart von var. tenuifolius hat, ebenso wie die erste, zwei große gleichschulterige Chromosomen (a). Die übrigen Chromosomen, auch zweischulterige, unterscheiden sich von den ersten durch eine geringere Dimension, obgleich die Korrelation der Schulterlänge bei ihnen auch variiert, aber die Korrelation der Schultern mit genügender Genauigkeit festzustellen ist unmöglich.

L. filicaulis (Abb. 18), der von einige Autoren als eine Abart von L. corniculatus betrachtet wird, wird karyologisch durch 12 Chromosomen charakterisiert, von welchen 2-ungleichschulterig (b), sich scharf durch die größten Dimensionen unterscheiden und 2 andere der Länge nach geringer (a) gleichschulterig sind. Die übrigen 8 Chromosomen sind am kleinsten und mehr oder weniger gleichschulterig.

Die folgende Gesamtart von L. angustissimus ist in unserer Untersuchung durch L. angustissimus und L. hispidus dargestellt. 
Wie bei der vorhergehenden Gesamtart haben die obenangewiesenen Arten eine Zahl von 12 Chromosomen. Bei L. angustissimus sind 2 Chromosomen ungleichschulterig, der Dimension nach klein (b), 4 Chromosomen (k) gleichschulterig, von ihnen ist ein Paar etwas größerer Dimension als das andere, die übrigen Chromosomen sind geringer, wahrscheinlicher sind sie zweischulterig. Diese Art unterscheidet sich von den anderen durch eine bedeutende Zahl von Chromosomen mit Trabanten. Auf der unten angeführten (Abb. 19) sind sie bei 4 Chromosomen vorhanden.

Der zweite Vertreter dieser Gesamtart L. hispidus (Abb. 20) hat eine doppelte Zahl von Chromosomen. Indem wir sein Idiogramm studieren, werden wir leicht 4 große ungleichschulterige Chromosomen (b) absondern, in Hinsicht auf die übrigen kann man nur eine Variierung der Schulterlänge und ein allmähliches Abnehmen der Chromosomengröße konstatieren. Eine weitere Gruppierung durchzuführen war unmöglich.

Die letzte von uns studierte Untergattung ist die Untergattung Tetragonolobus. Die Zahl der Chromosomen bei allen ihren von uns studierten Vertretern ist 14.

Die Segmentation der Chromosomen bei L. siliquosus L. (Abb. 21) ist in unseren Präparaten schwach ausgedrückt, infolgedessen können wir nur 4 der gröBten Chromosomen (a) absondern, deren Schulterkorrelation $1: 1$ ist und 4 ungleichschulterige Chromosomen (b).

Von 14 Chromosomen des Tetragonolobus (Abb. 22) sind 10 Chromosomen gleichschulterig und 4 mit Köpfchen (d). Der Größe nach müssen sie alle zu den größeren Chromosomen zugerechnet werden. Ihre Größe variiert bedeutend weniger, als bei den anderen Arten der Gattung.

Die Chromosomen von L. Requieni (Abb. 23) sind der Größe nach verschieden, aber eine Grenze zwischen den großen, mittleren und kleinen, infolge der fließenden Übergänge zu durchführen, ist sehr schwierig. Es ist bequemer sie auf Grund der Korrelation der Schulterlänge $\mathrm{zu}$ gruppieren -4 Chromosomen sind ungleichschulterig (d), ihre kurze Schulter ist so klein, daß sie als ein Köpfchen angesehen werden kann, und 4-gleichschulterige Chromosomen (a). Bei den übrigen ist die Schulterkorrelation schwierig zu bestimmen.

Zum Zwecke einer größeren Anschaulichkeit-Bequemlichkeit beim Zusammenstellen und Illustrieren unserer Folgerungen sind die erhaltenen karyologischen Ergebnisse über Tribus Loteae, Gattung Lotus in der untenangeführten Tabelle 3 dargestellt. 


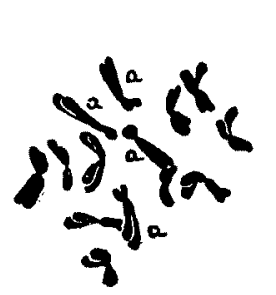

12

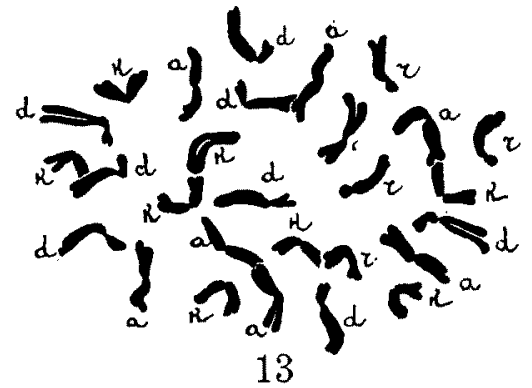

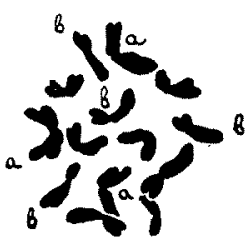

21

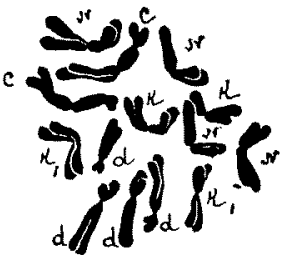

14

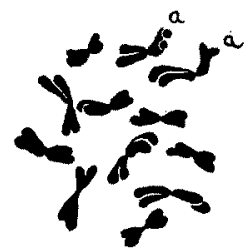

17

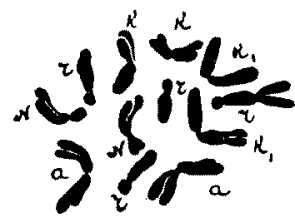

15

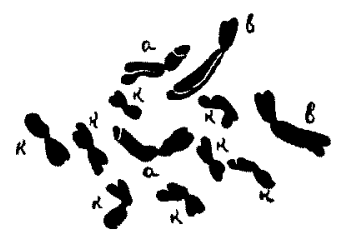

18

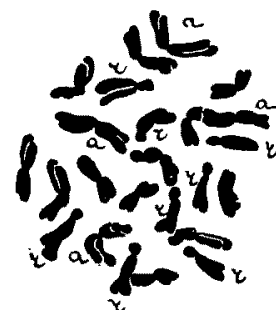

16

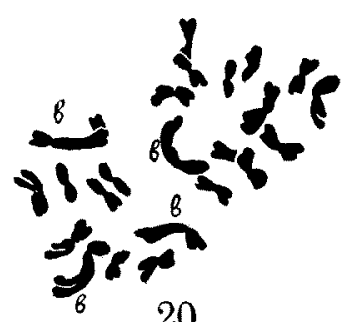

20

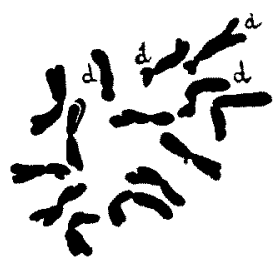

22

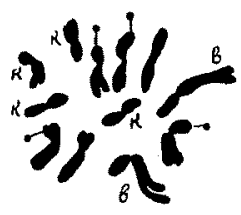

19

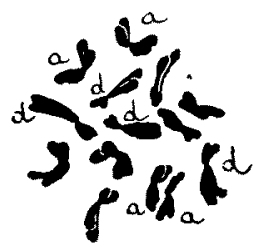

23

Abb. $12-23$

12. Lotus ornithopodioides L.

13. L. Creticus L.

14. L. cytisoides L.

15. L. uliginosus SchkuнR.

16. L. corniculatus v. alpestris LAMotTE.

17. L. corniculatus v. tenuifolius $\mathrm{L}$.
18. L. filicaulis $\mathrm{L}$.

19. L. angustissimus L.

20. L. hispidus DESF.

21. L. siliguosus L.

22. L. Tetragonolobus L.

23. L. Requienii. 


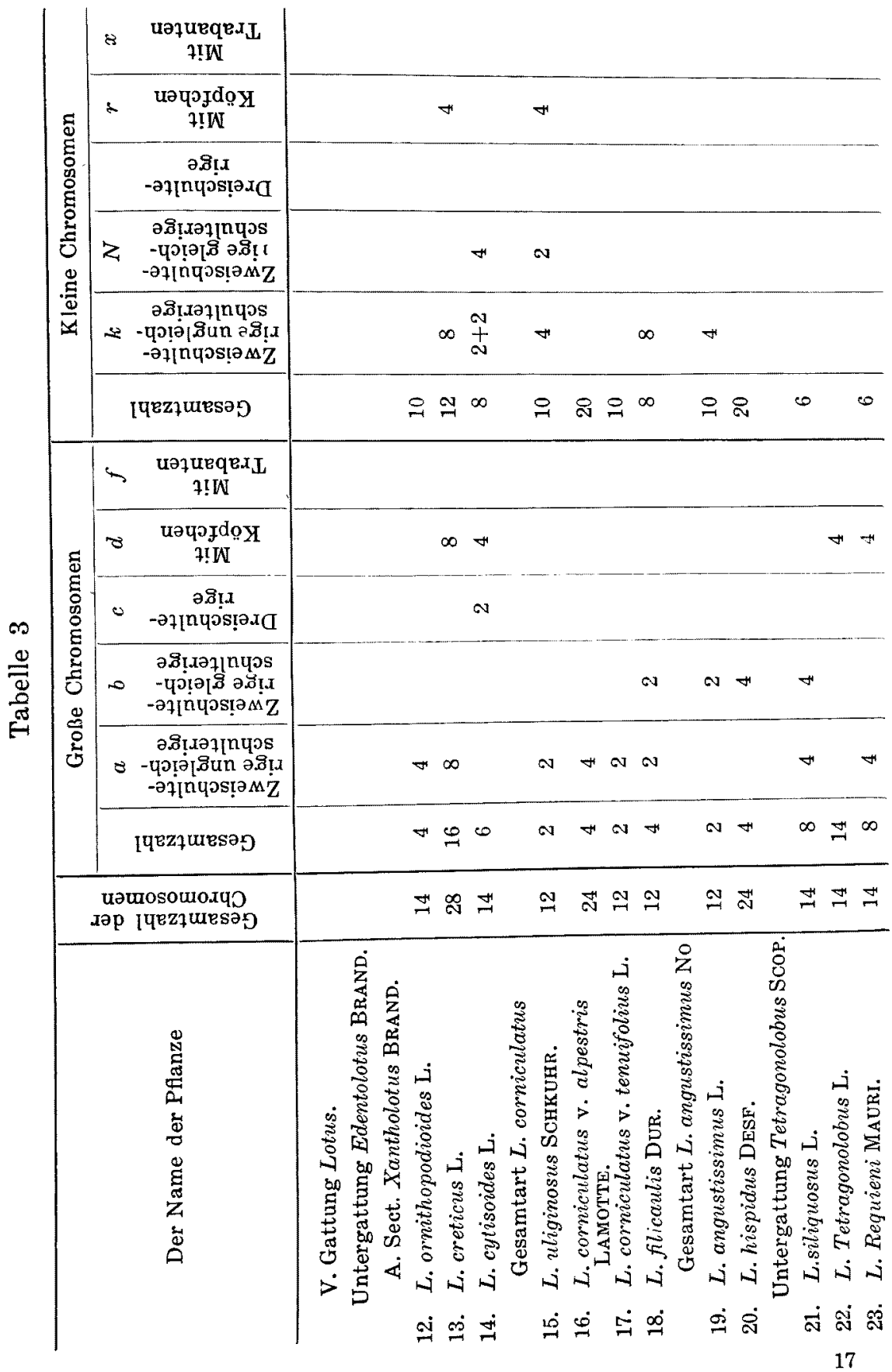


Auf Grund der von uns erhaltenen Ergebnisse sehen wir, daß die Chromosomenzahlen nicht als genügende Charakteristik für einzelne Gattungen dienen können. So z.B. in der Gattung Anthyllis finden wir Zahlen von 14 und 16 Chromosomen, in der Gattung Lotus 12 und 14. Aber wenn wir die von uns studierten Arten nach Sektionen und Sammelarten betrachten, so charakterisieren sich einige Sektionen und Sammelarten durch eine vollkommen bestimmte $\mathrm{Zahl}$ von Chromosomen.

In der Gattung Lotus treffen wir außer Diploid-auch Tetraploidarten, ähnliche Fälle werden sogar innerhalb einer Art beobachtet. So z.B. bei L. corniculatus v. tenuifolius, sind 12 Chromosomen, aber bei L. corniculatus var. alpestris-24. Man muß vermuten, daß die erste eine Ausgangs- und die zweite eine abgeleitete Form ist.

Die phylogenetische Nähe dieser Abarten wird nicht nur durch ein und dasselbe Vielfache der Chromosomen (12) bestätigt, sondern auch durch ihre Größe und Form. Während bei $L$. corniculatus var. tenuifolius 2 große zweigleichschulterige Chromosomen sind, sind bei v. alpestris 4 Chromosomen, die Gesamtzahl der mittleren und kleinen Chromosomen ist bei der ersten Abart 10, bei der zweiten 20.

Die karyologischen Ergebnisse geben uns die Möglichkeit Lotus angustissimus (12 Chr.) auch als Ausgangsart, aber L. hispidus (24 Chr.) als abgeleitete Art zu rechnen. Diese beiden Arten gehören der Sammelart $L$. angustissimus an. Die erste von ihnen hat zwei zweiungleichschulterige und 10 mittlere und kleine Chromosomen, die zweite-4 zweiungleichschulterige, aber 20 mittlere und kleinere Chromosomen.

Indem wir die Chromosomenzahlen von $L$. cytisoides und $L$. creticus zusammenstellen, bemerken wir auch, daßs eine Form diploid und die andere tetraploid ist. Infolgedessen konnte man $L$. cytisoides als Ausgangs- und $L$. creticus als abgeleitete Form betrachten. Bei genauerem Studieren der Idiogramme dieser Arten, trotz der Anweisungen einiger Systematiker, die L. cytisoides als eine Abart von L. creticus betrachten, sehen wir, daß nach den karyologischen Kennzeichen diese Arten nicht verwandt sind.

Ohne die oben angeführte Charakteristik der Idiogramme der einen und der anderen Art zu wiederholen, muß dennoch folgendes angemerkt werden.

Typisch und allgemein für beide Arten ist das Vorhandensein genügend großer der Dimension nach, ungleichschulteriger Chromosomen, bei welchen die kurze Schulter so klein ist, daß bei einer 
gewissen Orientierung sie sich der Dimension nach, dem Köpfchen nähert. Von diesen Chromosomen hat L. cytisoides 4, L. creticus 8. In Hinsicht der übrigen Chromosomen der Größe und der Form nach, wird nur ein Unterschied beobachtet.

Es fehlen die bei $L$. cytisoides und $L$. creticus vorhandenen großen dreischulterigen Chromosomen. L. creticus hat 4 kleine Chromosomen, mit der Größe nach unbedeutenden Köpfchen, welche bei $L$. cytisoides nicht vertreten sind.

Weiter sind bei L. creticus 16 zweischulterige, gleichschulterige Chromosomen (sie zerfallen der Größe nach in 2 Gruppen $8+8$ ), bei $L$. cytisoides werden nur $4(2+2)$ gefunden. Endlich bei L. cytisoides sind anstatt 4 ungleichschulterigen Chromosomen, wie man vermuten muß, indem man diese Form als Ausgangsform betrachtet-8 Chromosomen.

Auf Grund des obenerwähnten, halten wir es für möglich eine Folgerung zu machen, daB wenn $L$. creticus als eine abgeleitete Form von L. cytisoides ist, so ist es notwendig nicht nur eine Verdoppelungserscheinung zuzulassen, sondern $\mathrm{da} B$ im Evolutionsprozesse weitere tiefere Veränderungen des Karyotypus stattgefunden haben, die Veränderungen der Größe und Form der Chromosomen herbeigeführt haben.

Es ist noch unwahrscheinlicher, daß L. creticus eine abgeleitete Art des von uns studierten $L$. ornithopodioides ist, da bei der ersten 8 große Chromosomen (mit Köpfchen) vorhanden sind, die bei der zweiten Art fehlen, weiter ist es bei L. ornithopodioides die Gesamtzahl der großen Chromosomen 4 und der kleineren 10 , bei $L$. creticus 16 große Chromosomen, 12 kleinere.

Es ist noch interessant, sich mit der Zusammenstellung der morphologischen Merkmale und Areale der oben betrachteten Diploid- und Tetraploidarten aufzuhalten.

L. angustissimus $(12 \mathrm{Chr}$.) und L. hispidus (24 Chr.) gehören $\mathrm{zu}$ einer und derselben Sektion. Bei der ersten von ihnen sind die Früchte 3-6 Mal länger als der Kelch, die Samen genügend groß, obgleich ihre Zahl unbedeutend ist (etwa 5), bei der zweiten Art sind die Früchte geringer, die Samen kleiner, aber ihre Zahl erreicht 12. Das Areal von L. angustissimus ist beträchtlich breiter, als bei $L$. hispidus.

Indem wir die von uns studierten Organismen, die zu den Abarten von L. corniculatus v. alpestris (24 Chr.) und v. tenuifolius $(12 \mathrm{Chr}$.) angehören, zusammenstellen, beobachten wir, daß beim ersten von ihnen die Höhe der Pflanzen, die Größe der Blumen und Früchte etwas größer ist, als beim zweiten, das Areal von v. alpestris können wir nicht 
genau feststellen, es gibt nur Angaben, daß die Unterart von eu-corniculatus, zu welcher v. alpestris angehört, eine Verbreitung wie die Art hat, während dem das Areal von v. tenuifolius kleiner ist.

Die obenangeführten Ergebnisse, und ebenfalls die Resultate der Zusammenstellung von $L$. creticus mit anderen studierten Arten, die zur Sektion Xantholotus angehören, können immerhin nicht als genügender Grund zur Feststellung dienen, dab Tetraploidarten und Abarten der Gattung Lotus sich von Diploidarten durch größere Dimensionen der vegetativen und generativen Organe unterscheiden.

Tribus Loteae ist nach einer ganzen Reihe von Kennzeichen der Tribus Trifolieae verwandt. Den morphologischen Merkmalen nach unterscheiden sie sich nur im Bau der vegetativen Organe, wobei eine Reihe von Zwischenformen die Möglichkeit entzieht zwischen ihnen scharfe Grenzen durchzuführen. Die Vertreter beider Tribuse sind im Gebiet des mittelländischen Meeres verbreitet (siehe Tabelle 1).

Tribus Trifolieae hat ein weiteres Areal und die Polyploidieerscheinung ist hier am größten (96 ca. 130). Für beide Tribus sind jedoch die Größen der Chromosomen und die Grundzahlen 14 und 16 gleich.

Aus dem obenerwähnten und aus den Tabellen 2 und 3 sieht man, da $B$ das Studium der individuellen Eigentümlichkeiten bei den kleinen Chromosomen auf eine Reihe Schwierigkeiten stößt, wir konnten nur einen Teil der Chromosomen und nicht den ganzen Satz individualisieren und infolgedessen sind unsere Charakteristiken kein erschöpfendes Idiogramm und haben einen fragmentarischen Charakter. Aber trotz der Unvollständigkeit der erhaltenen Ergebnisse konnten wir sie doch bei der Entscheidung der Frage der phylogenetischen gegenseitigen Beziehungen zwischen den Diploid- und Tetraploid-Arten ausnutzen.

\section{Tribus Phaseoleae BRONN.}

Im Gegensatz zu Tribus Loteae, der noch von niemand zytologisch studiert ist, ist Tribus Phaseoleae ein Forschungsgegenstand von einer Reihe von Autoren gewesen. (KaRPETSCHENKo, WeINSTEIN, KaChIDSE, MuTO, RAU).

Die Forschung umfaßt 4 Gattungen und etwa 33 Arten. Trotzdem haben wir diese Tribus in's Programm unserer Arbeit eingeschloßen, haben das ganze bei uns vorliegende Material ausgenutzt und die Resultate unserer Fcrschungen haben uns zum Beschluß geführt, daß diese Gruppe der Bohnenarten, karyologisch weit nicht so einförmig ist, wie man auf Grund der vorliegenden Arbeiten der obenangewiesenen Forscher denken kann. 
Wir hoffen, daß bei der Mithilfe der Botaniker und der botanischen Gärten verschiedener Länder, es uns gelingen wird, Samen von noch nicht untersuchten Gattungen zu erhalten und unsere Untersuchung fortzusetzen.

Tribus Phaseoleae interessiert uns noch dadurch, daß nach den Ergebnissen von KARPETSCHENKo bei den Bohnen ziemlich oft eine Syndiploidieerscheinung beobachtet wird, und wir wollten den Verbreitungsgrad dieser Erscheinung bei den andern Vertretern der obenerwähnten Tribus bestimmen.

Tribus Phaseoleae schließt eine beträchtliche Zahl von Gattungen ein. Nach den Ergebnissen von CaPITAINe teilt TaUbert die Tribus Phaseoleae in folgende Subtribus ein :

1) Glycininae (12 Gattungen und 129 Arten, von uns sind 2 studiert. Clitoria $-16 \mathrm{Chr}$. und Glycine-20 Chr.)

2) Erythrinae (7 Gatturgen, 77 Arten; die Gattung Erytrinaeaca. $44 \mathrm{Chr}$. ist von uns studiert).

3) Galactiinae (6 Gattungen, 69 Arten).

4) Diocleinae (6 Gattungen, 60 Arten; Canavalia-22 Chr. ist studiert).

5) Cajaninae (8 Gattungen, 224 Arten; studiert ist die Gattung Rhynchosia-22-24 Chr.)

6) Phaseolinae (8 Gattungen, 232 Arten ; st:diert sind Phaseolus$22 \mathrm{Chr}$., Vigna-22 und Dolichos-22 Chr.)

Wie aus der obenangeführten Tabelle $1 \mathrm{zu}$ ersehen ist, sind die Vertreter der von uns studierten Tribus, im Gegensatz zu Loteae, Trifolieae die Bewohner der tropischen Länder, werden in Asien, Afrika und Amerika getroffen, erreichen die größte Verbreitung und Anzahl von Arten in Süd-Asien (115).

Nach den morphologischen Merkmalen nähert sich diese Tribus der Tribus Vicieae, untercheidet sie sich von ihr durch die Abwesenheit der Nebenblätter, die mittlere Blattader geht richt ins Stächlein und in die Ranke über, die Blätter sind meistenteils 3 zählig (gefiedert), der Fruchtknoten ist an der Basis von einer Scheibe umringt.

Nach den Ergebnissen der Karyologie sind alle früher studierten Gattungen der Chromosomengröße nach, gleich, jedoch werden Unterschiede bezüglich der Chromosomenzahlen beobachtet. Ungleiche Chromosomenzahlen (22 und 24), die von verschiedenen Forschern, nicht nur für verschiedene Arten einer Gattung, sondern auch für eine und dieselbe Art angeführt werden, veranlassen uns zuzulassen, daß diese Unterschiede wahrscheinlich von der Verschiedenheit in der Berechnung verschiedener Forscher abhängen. 
Der Grund der ausgesprochenen Vermutung ist folgender: KARPETSCHENKo führt für alle (11) von ihm studierten Arten der Gattung Phaseolus, Vigna und Dolichos eine Chromosomenzahl von $2 \mathrm{n}=22$ an. RAU führt für alle (5) von ihm studierten Arten derselben Gattungen eine Chromosomenzahl von $2 n=24$, an.

Unseren Forschungen gemäß, auf Grund der von uns studierten 9 Arten, die zu den obenangewiesenen drei Gattungen gehören, bleibt die Zahl der Chromosomen $2 \mathrm{n}=22$ beständig; von den andern Forschern weist nur NĚmec (1910a) für Dioclea (=Dolichos) multiflora 24 Chromosomen an, die übrigen-KATAYAMA (1928), LEWITSKy (1929) für Phaseolus radiatus, Muto (1929) für $P$. chrysanthosa, WEINSTEIN (1926) für $P$. vulgaris, KACHIDSE (1925) für $D$. Lablab führen $2 \mathrm{n}=22$ Chromosomen an.

$A u B e r d e m$, in den Fällen, wenn eine und dieselbe Art von mehreren Autoren studiert wurde, stimmen die Ergebnisse von RAU mit den Ergebnissen der anderen Forscher nicht überein, so z.B. hat nach Katayama Phaseolus radiatus $22 \mathrm{Chr}$. nach Lewitsky $22 \mathrm{Chr}$., aber nach RAU $24 \mathrm{Chr}$. Dolichos Lablab hat nach KARPETSCHENKo $22 \mathrm{Chr}$., nach Katayama $22 \mathrm{Chr}$, aber nach RAU $24 \mathrm{Chr}$. Die Beständigkeit der Zahlen, die von RAU 24 und von KARPETSCHENKo 22 angeführt werden, ist, wie es scheint, für uns eine Hinweisung, daß es sich hier nicht um eine Variation der Zahlen bei verschiedenen Arten einer Gattung und sogar in den Grenzen einer Art, sondern um eine Verschiedenheit beim Berechnen handelt.

Auf Grund unserer Untersuchungen, scheint uns die Zahl von 22 Chromosomen richtiger, wir führen diese Zahl für alle von uns studierten Arten der Gattung Phaseolus, Vigna, Dolichos an.

Für Erythrina Crista Galli (Abb. 26) ist die Zahl von 44 Chromosomen am richtigsten. Diese Art ist im Vergleich mit den anderen 22 Chromosomen tetraploid. Abhängig davon, ob wir ihre Chromosomen als scharf zweischulterig, oder als vier, die sich mit ihren Enden nähern, rechnen, schwankt ihre Zahl von 42 bis 44 . Auf unserer Abbildung 26 sind 43 Chromosomen; es ist möglich, daB die obenangewiesenen Schwierigkeiten als Ursache der obenerwähnten Verschiedenheit bei Berechnung gedient haben. RAU zählt anstatt 22,-24 Chromosomen. Aber YaMAHA und SinoTô (1925) führen anstatt 40 für Glycine Soja var. Akasaya 38 an. Wir stießen mit ähnlichen Schwierigkeiten beim Studieren von Rhynchosia phaseoloides zusammen; die Chromosomen konnten wie 22 , so auch wie 24 gezählt werden. Eine wahrscheinlichere Zahl ist 22, aber bis zur endgültigen Aufklärung der Chromosomenzahl enthalten wir uns eine Abbildung dieser Gattung zu geben. 
Bei $P$. nigerinus (Abb. 30) und P. capensis (Abb. 31), die bisher noch von niemand untersucht wurden, ist die Chromosomenzahl auch 22. Dieselbe Chromosomenzahl ist von KARPETSCHENKo auch bei Vigna uniguiculata (22) und $V$. Catjang und von uns-bei V. vexillata (Abb. 32), V. glabra (Abb. 33) und V. owahnuensis (Abb. 34) gefunden.

Indem wir unsere Forschung auf andere Gattungen dieser Tribus Rhynchosia und Canavalia verbreiteten, fanden wir die Chromosomenzahl 22. Also aus der von uns studierten Gattungenzahl der Tribus Phaseoleae charakterisieren sich durch eine Chromosomenzahl 22 die Gattungen Phaseolus, Vigna, Dolichos, Canavalia, Rhinchosia. Bei anderen Gattungen Glycine (G. hispida und G. ussuriensis) und Erythrina (E. Crista Galli) sind $2 \mathrm{n}=40$ Chromosomen.

Der Größe und Form nach sind die Chromosomen bei allen von uns studierten Arten der obenangewiesenen Gattungen gleich, ihre Größen sind unbedeutend und die individuellen Eigenheiten festzustellen war nicht möglich. Durch Zahl und Größe unterscheiden sie sich scharf von den Idiogrammen der Vertreter der Tribus Vicieae.

Bei der Untersuchung von Clitoria ternatea fanden wir einen eigenartigen und ganz neuen Chromosomensatz für die Tribus Phaseoleae. Vor allem ist ihre Zahl weder 20 noch 22, aber 16. Die Chromosomengröße ist viel bedeutender und der Größe nach sind die Chromosomen nicht gleich. Der Unterschied der Größe nach ist so groß,

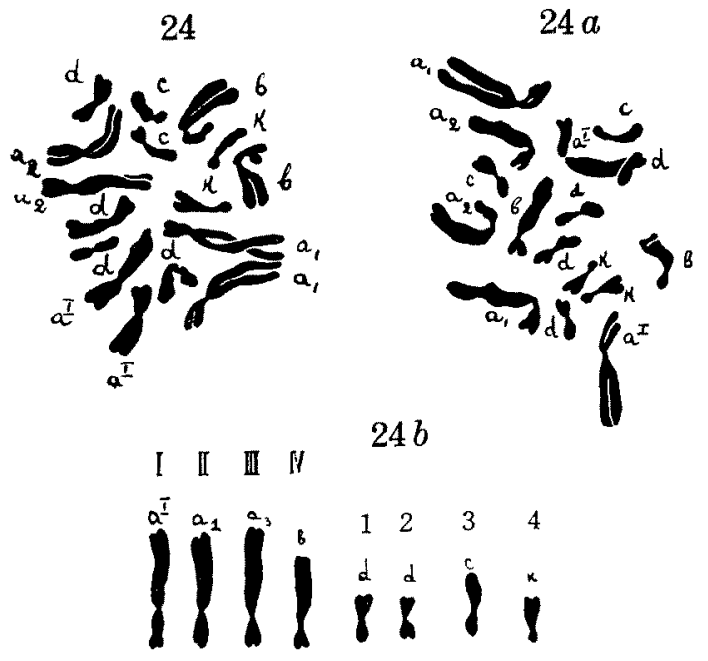

Abb. 24, 24a, 24b. Clitoria ternatea daß man den Eindruck erhält, daß das Idiogramm dieser Art zwei verschiedene Chromosomensätze einschließt, 8 große und 8 kleinere Chromosomen.

Ein ausführlicheres Studium zeigt, daß die Chromosomen jeder dieser Gruppen auch ungleich sind. Ein von den vier Paaren grober Chromosomen (b) ist kleiner als die übrigen.

In der Korrelation der Schultern wird auch ein Unterschied beobachtet, obgleich alle vier Paar der 


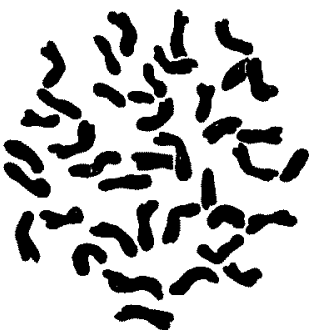

25

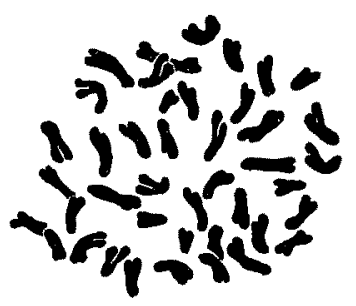

26

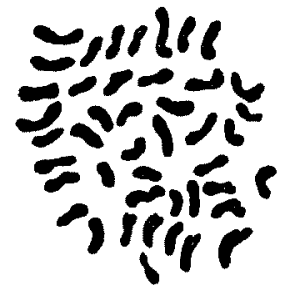

27

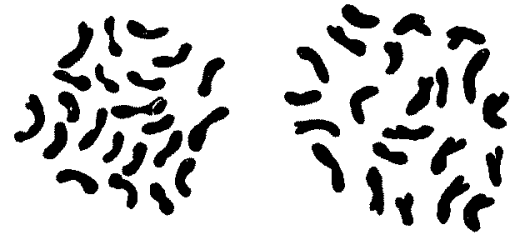

28

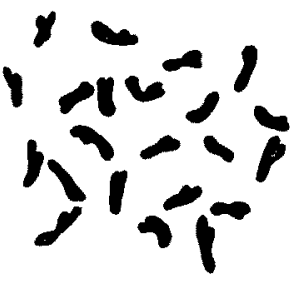

30

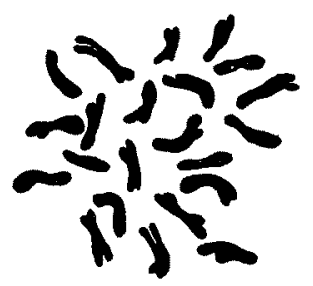

34

29

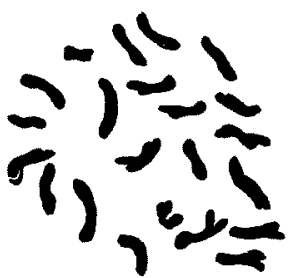

32

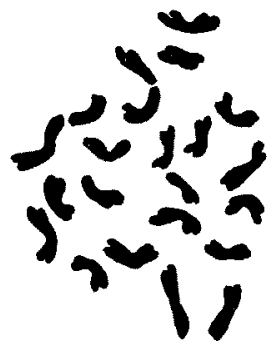

35

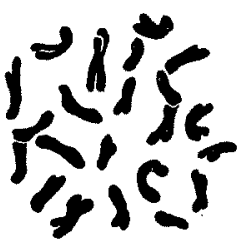

31

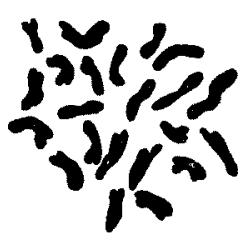

36

Abb. 25-37

25. Glycine ussuriensis

26. Erythrina Crista Galli L.

27. \} Canavalia gladiata DC.

29. Phaseolus aconitifolius JACQ.

30. $P$. nigerinus J ASS.

31. P. capensis THUNB.
32. Vigna vexillata BENTH.

33. V. glabra SAVI.

34. V. owahnuensis VoG.

35. Dolichos Lubia Forsck.

36. D. niloticus DEL.

37. D. ornatus WALL. 
großen Chromosomen auch ungleichschulterig sind, aber die Größen der kurzen Schulter sind ungleich, sie ist am größten bei den Chromosomen I und am kleinsten bei den Chromosomen IV; die Chromosomen II und III sind in Beziehung der kurzen Schulter ein Übergang von der I zum IV Chromosome (Abb. 24, 24a, 24b).

Die zweite Gruppe-8 kleinere Chromosomen ist ihrer Form nach im bedeutenden Grade mit den früher betrachteten Chromosomen analogisch, auch hier ist die kurze Schulter ungleich, sie ist am größten bei dem I-ten Chromosome und am kleinsten bei dem IV-ten, das II-te Chromosom bildet auch hier, bezüglich der kurzen Schulter einen Übergang $z$ wischen dem I-ten und dem IV-ten.

Eine Analogiestörung zwischen der Gruppe großer und kleinerer Chromosomen ist, daß bei Chromosomen I und II (scharf ungleichschulterig) die Korrelation der Schulter sich zu 1:3 nähert, die Chromosomen 1 und 2 sind beinahe gleichschulterig. Clitoria ternatea verletzt die karyologische Einförmigkeit der Tribus Phaseoleae; ein scharfer Unterschied von den anderen Arten der Idiogramme nach veranlabt uns zu vermuten, $\mathrm{da} B$ wir bei weiterer Untersuchung dieser Grupre noch aus neue Überraschungen stoßen können. Vollkommen möglich ist, da $\beta$ der Abgrund, welcher zwischen dieser und den Nachbartribus existierte, im gewissen Grade durch unsere geringen Kenntnisse über die Karyologie dieser Tribus erklärt wird. Von 65 Gattungen sind heutzutage nur 8 studiert, ein sehr geringes $\% 12.3$.

Jedenfalls unterscheidet sich Clitoria ternatea karyologisch bedeutend von den anderen früher studierten Gattungen und Arten der Tribus Phaseoleae.

Den morphologischen Kennzeichen nach, finden wir keine so scharfe Unterschiede. Indem wir Clitoria und Glycine (beide Gattungen gehören der Subtribus Glycininae an) vergleichen, wird nur ein Unterschied in der Gebärtung des Griffels längs der Innenseite und der Blumenzahl im Blütenstand beobachtet.

Indem wir unsere Untersuchung beendigen, muß noch die Frage über die Syndiploidie in der Tribus Phaseoleae berührt werden. Wie oben erwähnt wurde, beobachtete KARPETSCHENKo diese Erscheinung bei Bohnen sehr oft.

In der Arbeit von Fukushima (Japanese Journal of Botany) gibt es eine Anweisung, daB Y. KAWAKAMI, der die Gartenabarten von Glycine Soja zytologisch studierte, oft in den Wurzelspitzen der Gruppe Tetraploidzellen zwischen Diploidzellen fand. Wir haben auch die Erscheinung der Synploidie bei Canavalix gladiata (Abb. 27 und 28) beobachtet. 
Tabelle 4

Karyologische Gesamtübersicht der Tribus Phaseoleae BronN.

Subtribus Glycininae TAUBERT

Gattung Clitoria L.

1. C. ternatea L. . . . . $16 \mathrm{Chr}$. Tschechow und Kartaschowa 1931 b.

Gattung Glycine L.

2. G. ussuriensis .... 40 Chr. Tschechow und Kartaschowa 1931 b.

3. G. Soja Sieb. et ZuCC. . $40 \mathrm{Chr}$. KARPETSChENKo $1925 \mathrm{~b}$, KawAKAMI

(=Soja hispida). $1930^{1)}$.

4. G. Soja var. Akasaya . . $38 \mathrm{Chr.} \mathrm{Yamaha} \mathrm{und} \mathrm{Sinotô} 1925$.

Subtribus Erythrineae TAUBERT

Gattung Erythrina

5. E. Crista Galli L. . . ca $40 \mathrm{Chr}$. Tschechow und KaRTaschowa 1931 b.

Subtribus Diocleinae TAUB.

Gattung Canavalia.

6. C. gladiata DC. . . . . $22 \mathrm{Chr}$. TSChEChOW und KaRtaschowa $1931 \mathrm{~b}$.

Subtribus Phaseolinea TAUBERT

Gattung Phaseolus TourN.

7. P. aconitifolius JacQ. . . $22 \mathrm{Chr}$. Tschechow und Kartaschowa $1931 \mathrm{~b}$.

8. P. actifolius A. Gray. . . $22 \mathrm{Chr}$. KarPETSChenko 1925.

9. P. angularis Willd. . . $22 \mathrm{Chr}$. KaRPETSCHENko 1925.

10. P. aureus Roxb. . . . $22 \mathrm{Chr}$. KARPETSCHENKo 1925.

11. P. capensis Thunb. . . 22 Chr. Tschechow und KaRTaschowa $1931 \mathrm{~b}$.

12. P. multiflorus WiLld. . $22 \mathrm{Chr}$. KaRPETSCHENKo $1925 \mathrm{~b}$.

13. P. chrysanthosa. . . $22 \mathrm{Chr}$. Muto 1929.

14. P. lunatus L. . . . . . . 22 Chr. KarPETSChenko 1925 b, KaWAKami, 1930.

15. P. Mungo L. . . . . 22 Chr. Karpetschenko 1925 b.

16. P. mungo . . . . . 24 Chr. RaU 1929 b.

17. P. nigerinus Juss. - . 22 Chr. Tschechow und Kartaschowa $1931 \mathrm{~b}$.

18. P. radiatus . . . . . 22 Chr. Katayama 1928, Lewitzky 1929.

24 Chr. RAU $1929 \mathrm{~b}$.

19. P. trilobus . . . . . $22 \mathrm{Chr}$. KARPETSCHENko $1925 \mathrm{~b}$.

2). P. vulgaris L. . . . . . $22 \mathrm{Chr}$. KARPETSCHENKO $1925 \mathrm{~b}$, WEINSTEIN 1926 ,

Gattung Vigna SAVI.

KAWAKAMI 1930.

21. V. Catjang WalP. . . . 24 Chr. Rau 1929 b, 22 Chr. Karpetschenko 1925 b.

22. V. glabra Savi. . . . . $22 \mathrm{Chr}$. Tschechow und Kartaschowa $1931 \mathrm{~b}$.

23. V. owahnuensis Vog. . . $22 \mathrm{Chr}$. TsCHeCHOW und KaRTaschowa $1931 \mathrm{~b}$.

24. V. ungiculata (L.) WALP. 22 Chr. KARPETSCHENKo 1925 b.

25. V. vexillata BENTH. . . . $22 \mathrm{Chr}$. Tschechow und Kartaschowa $1913 \mathrm{~b}$.

Gattung Dolichos L.

26. D. biflorus. . . . . . $24 \mathrm{Chr}$. RAU $1929 \mathrm{~b}$.

27. D. Lablab L. . . . . . 22 Chr. KARPETSCHENKO 1925b, KaCHIDSE 1925, 24 Chr. RAU 1929 b.

28. D. Lubia Forsck. . . . . $22 \mathrm{Chr}$. Tschechow und Kartaschowa 1931 b.

29. D. multiflora. . . . . $24 \mathrm{Chr}$. NĚmec 1910 a.

30. D. niloticus Dol. . . . $22 \mathrm{Chr}$. Tschechow und Kartaschowa $1931 \mathrm{~b}$.

31. D. ornatus WALL. . . . $22 \mathrm{Chr}$. Tschechow und Kartaschowa $1931 \mathrm{~b}$.

Gattung Rhynchosia Lour.

32. R. phaseoloides DC. . . 22 Chr. Tschechow und Kartaschowa 1931 b.

1) Ref. in der Zeitschrift f. Pflanzenziuchtung, 1931, Bd. XVI, H. 3, S. 513: KawAkAM I, J., Chromosome numbers in Leguminosae. Bot. Mag., Tokyo, 44. S. 319 $328,1930$. 


\section{Verzeichnis der Pflanzennamen mit den Nummern der entsprechenden Zeichnungen und dem Herkunftsort der Samen in Klammern.}

1) Anthyllis maritima Schweigg. . 1 (Botanischer Garten der Universität zu Warschaua, Polen)

2) A. alpestris Kıт. . . . . . . . 2 (Zürich)

3) A. Barba Jovis L. . . . . . . 3 (Botanischer Garten der Universität von Coimbra, Portugal)

4) A. Gerardi L. . . . . . . . 4 (Botanischer Garten in Brno, Tschechoslovakei)

5) A. tetraphylla L. . . . . . . 5 (Botanischer Garten in Brno, Tschechoslovakei)

6) Hymenocarpos circinnatus SAvI. 6 (Woronesh)

7) Securigera coronilla DC. . . . . 7 (Nikitischer Botanischer Garten, Krim)

8) Dorycnium hirsutum Ser. . . . 8 (Urbino, Italien)

9) D. rectum SER. . . . . . . . . 9 (Modena, Italien)

10) D. suffruticosum VILL. . . . . 10

(Botanicher Garten, Marburg)

11) D. herbaceum VILL. . . . . . . 11

(Botanicher Garten, Marburg)

12) Lotus ornithopodioides L. . . . 12 (Delft Holland)

13) L. creticus L. . . . . . . . 13 (Botanischer Garten der. Universität zu Coimbra, Portugal)

14) L. cytisoides L. . . . . . . 14

(Botanischer Garten der Universităt zu Coimbra, Portugal)

15) L. uliginosus SCHKUHR. . . . 15 (Botanischer Garten der Universität zu Coimbra, Portugal)

16) L. corniculatus var. alpestris LAmotTe. (Oesterreich) . . . . 16

17) L. corniculatus var. tenuifolius L. 17 (Botanischer Garten der Universität zu Warschau, Polen)
18) L. filicaulis Dur. . . . . . . . 18 (Botanischer Garten Hauniensis)

19) L. angustissimus L. . . . . . . 19 (Befindungsort unbekannt)

20) L. hispidus DesF. . . . . . . 20 (Woronesh)

21) L. siliquosus L. . . . . . . . . 21 (Kowno)

22) L. Tetragonolobus L. . . . . 22 (Kowno)

23) L. Requieni Mauri. . . . . . . 23 (Befindungsort unbekannt)

24) Clitoria ternatea L. . . 24, $24 \mathrm{a}, 24 \mathrm{~b}$ (Botanischer Garten, Marburg)

25) Glycine ussuriensis. . . . . 25 (Botanisches Kabinet des SüdUssurischen Gebietes StaatsRussische Geographische Abteilung)

26) Erythrina Crista Galli L. . . . 26 (Lissabon)

27) Canavalia gladiata DC. . . . 27,28 (Botanischer Garten, Marburg)

28) Phaseolus aconitifolius JACQ. . . 29 (Botanischer Garten, Bukarest)

29) P. nigerinus JAss. . . . . . . 30 (Botanischer Garten, Bukarest)

30) P. capensis ThuB. . . . . . . 31 (Botanischer Garten Bukarest)

31) Vigna vexillata BenTH. . . . . . 32 (Zìrich)

32) V.glabra SAvi. . . . . . . 33 (Zürich)

33) V. owahnuensis VoG. . . . . . 34 (Lissabon)

34) Dolichos Lubia Forsk. . . . . . 35 (Botanischer Garten, Bukarest)

35) D. niloticus DEL. . . . . . . 36 (Botanischer Garten, Bukarest)

36) D. ornatus WALL. . . . . . 37 (Botanischer Garten, Bukarest) 


\section{Literatur}

1. Avdulow, N.P., 1931. Karyo-systematische Untersuchung der Familie Gramineae. Akademie d. landwirts. Wiss. Namens Lenin Institut d. Pflanzenzucht Leningrad.

2. Ascherson u. Graebner, 1908. Synopsis der mitteleuropäischen Flora 6. Abt. 2. (1908-13).

3. Bleier, H., 1925. Chromosomenstudien bei der Gattung Trifolium. Jahrb. f. wiss. Bot. 68, 604-636 (1925).

4. Brand, A., 1898. Monographie der Gattung Lotus. Bot. Jahrb. f. Systematik, Pflanzengesch. u. Pflanzengeogr. Bd. 25.

5. Capitaine, 1913. Analytisches und phytogeographiches Studium der Gruppe Leguminosae. Geograph. bot. Ber. Bd. 23.

6. Delaunay, L.N., 1915. Tetraploidindividuum von Muscari, Latifolium. Protokoll d. Tagung d. Naturforscher-Gesells.

7. - 1915. Vergleichende Karyologische Untersuchung der Arten Muscari. Abh. d. Naturforscher-Gesells. von Kiew.

8. - 1922. Vergleichende karyologische Forschung der Arten Muscari MILL und Bellevalia LAPEYR. Anzeiger d. bot. Gart. von Tiflis Ser. II. Ausg. 1.

9. - 1925. Chromosomen bei den Arten Ornithogalum L. Abh. des staatswissenschaftlichen Forschungsinstituts Namens Timiriasew Ser. 1. Abt. II. Ausg. 3.

10. - 1926a. Verwandlung des Zellkernes in Erbveränderlichkeit. Abhandlungen. S. G. Botanik 1 .

11. - 1926. Chromosomentheorie der Vererbung und Chromosomen bei einigen Lilienarten. Anzeiger d. bot. Gar. von Tiflis, Ser. II. Ausgabe 2.

12. - 1929. Kern und Art. 1. Typische Chromosomenformen. Planta 7.

13. Dombrowskaya-Slutskaya, L. N., 1927. Somatische Teilung des Kernes bei Cicer orietinum. Zeits. d. russis, bot. Ges., B. 12, Nr. 1-2.

14. Engler, A. u. Prantl, K., 1894. Die natürlichen Pflanzenfamilien III. 3. Leguminosae Taubert.

15. Ghimpu, V., 1928. Beitrag zur karyologischen Untersuchung der Gattung Medicago. C. K. Akad. der Wissenschaft, Paris 187. No. 4, 25-27.

16. - 1929. Über die Chromosomen von Vitis Medicago und Hordeum. Auszug d. Ber. d. Vereins d. Anat. Bordeaux 25-27.

17. - 1929. Über Karyologie der.Gattung Medicago. Auszug d. Landwirtschaftsber. No. $5-6$.

18. Gromowa, T., 1915. Bestimmer der Arten und Abarten der Gattung Lotus, die im Europäischen Russland und im Kaukasus gefunden werden. Das Schema ihrer Verbreitung. Abh. d. Bureau d. angewand. Botanik. Petrograd. 8. Jahr.

19. Hegi, G., 1925. Illustrierte Flora von Mittel-Europa Bd. IV. 3.

20. Karpetschenko, G. D., 1925. Utber Chromosomen der Bohnenarten. Abh. d. angew. Botanik u. Selektion XIV.

21. - 1925. Karyologische Skizze der Gattung Trifolium. Abh. d, angewand. Botanik u. Selektion 14.

22. - 1927. Polyploide Hybriden Raphanus sativus L. $\times$ Brassica oleracea L. Abh. d. angew. Botanik u. Selektion 17.

23. Kreuter, E., 1929. Chromosomenstudien bei den Galegeen. Ber. deut. bot. Ges. 47, 99-101.

24. - 1930. Beitrag zu karyologisch-systematischen Studien an Galegeen. Planta 2, Ht. 1 .

25. Lewitzky, G. A., 1924. Materialbasis der Vererbung. 
26. Lewitzky, G. A., 1926. Karyo-genotypische Veränderungen im Evolutionsprozesse. Abh. d. angew. Botanik u. Selektion 15.

27. Lewitzky, G. A., u. Kusmina, N. E., 1927. Karyologische Methode in Systematik und Phylogenetik der Gattung Festuca. Abh. d. angew. Botanik u. Selektion 16.

28. Muto, A., 1929. Meiotische Teilungen in Pollenmutterzellen von Phaseolus chrysanthos SAv. und Cassia occidentalis L. Abh. d. Kais. Univ. zu Kyoto S. B. 4, 265-271.

29. Nawaschin, M. S., 1915. Haploid-Diploid- und Periploidkerne bei Crepis viriens VILL. West. - Naturforscher-Gesells. Kiew I. 25.

30. Rollow, A. A., 1908. Wildwachsende Pflanzen des Kaukasus.-Tiflis.

31. Schürchow, P. N., 1926. Zytologie der Blütenpflanzen.

32. Senjaninowa-Kortschagina, 1929. Karyosystematische Untersuchung der Gattung Aegilops L. Abh. der Kongr. d. U.d. S. S. R. über Genetik u. Selektion. Leningrad. 10-16. Jan. B. II.

33. Sweschnikowa, J., 1927. Karyologische Studien an Vicia. Ber.d.angew, Botanik u. Pflanzenzucht 17., 37-72.

34. Tischler, G., 1921-1922. Allgemeine Pflanzenkaryologie. Berlin.

35. - 1927. Tabulae Biologicae. Bd. IV. Berlin.

36. Tschechow, WI., 1930. Karyologisch-systematische Untersuchung der Tribus Galegeae. Fam. Leguminosae. Planta 9. Ht. 4.

37. - 1931. Karyslogisch-systematische Untersuchung der Tribus Sophoreae, Podalarieae und Genisteae. Nachr. d. Russisch-Staatsbot. Ges. zu Tomsk.

38. Weber, C., 1913. Landwirtschaft. Jahrb. 44. Ht. 1-2. Berlin.

39. Werner, H., 1907. Handbuch des Futterbaues, Berlin. 